M. N. SAHA: Primary cosmic rays consist of proton and helium particles mainly, but neon particles are also found to occur quite strongly. Now the solar spectrum does not show the slightest trace of any lines of neon, though these occur in a very favourable region (red). So we have to admit that neon does not occur in the sun. How are we then to account for the occurrence of neon-nuclei in the primary cosmic-ray spectra?

H. AlfVÉn: The origin of cosmic-ray radiation is a complicated phenomenon, and a full discussion would take us too far now. The direct connexion between flares and increases in cosmic radiation indicates that at least part of the cosmic radiation is accelerated near the Sun. But it is an open question whether all the cosmic radiation originates near the Sun.

R. Q. Twiss: Will Prof. Alfvén comment on the time delay between the occurrence of the flare and the increase in cosmic-ray intensity at the Earth?

H. Alfvén: If the excess cosmic-ray flux is produced at the same time as the optical flare, one must attribute the delay to irregular magnetic fields between the Sun and the Earth which increase the effective path for charged particles with a factor $\sim 3-$ ro. Alternatively, if the excess is produced by a magnetic acceleration process following the flare, the delay could be attributed to the finite time needed for the magnetic energy to build up.

M. Minnaert : I believe that balloon observations during flares have indicated increases of cosmic radiation of the order of $200 \%$.

H. Alfvén: Yes. Upon the whole, the increases are more pronounced for smaller energies, such as registered by neutron counter and in balloon measurements.

M. Minnaert: Balloon measurements during flares are clearly important.

A. SCHLÜTER: Generation of cosmic radiation on the Sun seems to be proved only in the case of four big increases in cosmic radiation. For all other cases ('small flare effects') the interpretation of the increases as modulation of the existing cosmic radiation is not excluded. A continuous transition from 'small' to large flare effects has not been established as yet. Assuming modulation, the contribution of the Sun to the total cosmic-ray flux would be very small.

H. Alfvén: Yes, there is a possibility that flares only release cosmic rays available in reservoirs near the Sun.

D. H. Menzel. With reference to Dr Saha's remark, I should say that the solar abundance of neon is very uncertain because of the unfavourable location of its fundamental lines.

K. O. KIEPENHEUER: It is dangerous to draw too far-reaching conclusions from connexions between flare observations and cosmic radiation. There are many complicated processes taking place in the vicinity of flares.

\title{
ro. HYPOTHESES ON THE ORIGIN OF FLARES
}

By A. UnsöLD

\section{Some Observational Characteristics}

A theory of flares should explain the following observations:

(a) The rise to peak intensity within $\sim 5 \mathrm{~min}$.

(b) The time of decay being independent of the time of rise; mostly $\sim \mathrm{r} \mathrm{hr}$.

(c) Bright flares occur mostly in spot groups of maximum development; they are frequently connected with bright flocculi or plages faculaires.

(d) Flares seem to vary between $\sim$ I sec. diameter and $\sim 5^{-15} \mathrm{~min}$. duration and 5000 millionth of a hemisphere and several hours duration.

(e) Flares may occur in quite different levels; Ellerman's 'bombs' are probably below the photosphere, limb flares in the prominence level. 


\section{The Discharge Theory by Giovanelli and Hoyle}

Since in brightening big flares no appreciable Doppler effects have been observed, it is tempting to explain flares as a sort of 'solar flashes'. But while a terrestrial cloud is an almost perfect isolator, the solar atmosphere is a highly conducting medium.

In an ionized gas the effective radius $\rho_{i}$ for collisions between an ion with charge $e Z$ and an electron with the kinetic energy $\frac{m}{2} v^{2}$ is determined by $\frac{Z e^{2}}{\rho_{i}} \approx \frac{m}{2} v^{2}$, i.e. decreases with increasing velocity. Giovanelli asks: Above which field strength $E_{\mathrm{c}}$ will a 'runaway' electron gain more and more energy? His answer is: $E_{c} \approx \mathrm{I} \cdot 5 \times 10^{-14} n_{c}$ volt $/ \mathrm{cm}$.

However, in the solar atmosphere there are $\mathrm{IO}^{3}$ to $\mathrm{IO}^{4}$ times more neutral hydrogen atoms than electrons. Recent theoretical and experimental work shows that $\mathrm{H}$ atoms have a large Ramsauer cross-section, for $5000^{\circ}$-electrons $\rho_{0}=3$ angstrom. Neutral hydrogen therefore reduces the conductivity considerably. Since on the Sun the hydrogen is not far from becoming ionized, we get another mechanism of breakdown which in general should become effective prior to that investigated hitherto: If some currents develop sufficient Joule heat so that the ionization of hydrogen is increased, we get a 'leader' with higher current density, again more heat . . ., i.e. a flash. For pressures of $p_{\sigma} \sim$ Io $^{3}$ dyne $\mathrm{cm} .^{-2}$ about $10^{-3}$ volt $\mathrm{cm}^{-1}$ are sufficient. With $\sigma \approx 2 \times$ Io $^{12}$ elst. C.G.S. units, $E=$ IO $^{-3}$ volt cm. ${ }^{-1}=3 \cdot 3 \times$ IO $^{-6}$ elst. C.G.S. we get a current density of $i=0.67 \times$ $\mathrm{ro}^{7}$ elst. C.G.S. $=2 \times 10^{-3} \mathrm{amp} . \mathrm{cm} .^{-2}$ and, in a layer $\sim 200 \mathrm{~km}$. high, an amount of Joule heat corresponding roughly to $\mathrm{r} \%$ of the solar flux of radiation. So far everything appears quite reasonable. The question, however, how the necessary electromagnetic fields can be produced or how the necessary electromagnetic energy can be released fast enough leads into great difficulties.

\section{Criticism of the Discharge Theory by Cowling, Piddington et al.}

(a) The current density $i$ is connected with the rot of the magnetic field $\vec{H}$ by the Maxwell equation $\operatorname{rot} \vec{H}=\frac{4 \pi}{c} i$. As to order of magnitude, $\operatorname{rot} \vec{H} \approx H / l$, where $H$ and $l$ are a characteristic field strength and length. An $i \approx 0.67 \times 10^{7}$ elst. C.G.S. cannot be obtained under any reasonable assumptions. Even $H \sim 2000$ gauss would require $l$ as small as $7 \mathrm{~km}$. The observationally more likely figures of $H \approx$ ro gauss and $l \approx 10,000 \mathrm{~km}$. would imply an utterly insufficient current density.

(b) According to Giovanelli's theory, flares should occur most likely when $d H / d t$ is large. In reality, however, most big flares are observed during maximum development of spot groups, i.e. when-at least for the spot field- $d H / d t=0$. Photoheliograms rather suggest that the highly turbulent conditions prevailing between the two main spots of a complex group are favourable for flares.

(c) Concerning the electrodynamics of flares we should further be aware that-according to Alfvén's criterion-we are in the realm of magneto-hydrodynamics. Giovanilli's original work, however, completely neglects all reactions! In a resting medium the diffusion of an electromagnetic field (best described by the vector potential $\vec{A}$ giving $\vec{H}=\operatorname{rot} \vec{A}$ ) is an extremely slow process. Allowing material motion the electromagnetic energy transfer generally is not much larger than the convective one. All these arguments make one feel rather sceptical against a purely electrodynamical theory of flares.

\section{Flares and 'plages faculaires'. Concluding Remarks}

Perhaps more insight into the energy transfer within flares can be obtained from a detailed study of faculae and plages which probably are a related-only weakerphenomenon. The analysis of the observed centre-limb variation of faculae shows that, compared with the normal photosphere, the higher layers $(\tau<0 \cdot 9)$ obtain more energy, the deeper layers less. Thus, there seems to exist an additional mode of energy transfer 
between $\tau$ about I and $\mathrm{O} \cdot \mathrm{I}$. Careful observation and analysis of the spectra of faculae might reveal more about that almost unexplored problem.

A deeper understanding of flares can probably be obtained only in connexion with the magneto-hydrodynamics of supersonic turbulence in plasms. Radio and cosmic-ray astronomy shows that in such plasms electric fields and corpuscular radiations with a wide range of energies are quite important. The production of cosmic rays on the Sun, e.g., requires fields of at least $O \cdot I$ volt $\mathrm{cm}^{-1}$, that is roo times stronger than those mentioned under 2 .

\section{SUMMING UP}

By M. G. J. MinnaerT

Since the available time is rather short, I shall only give a very brief summary in the form of a diagram, which may be a basis for discussion.

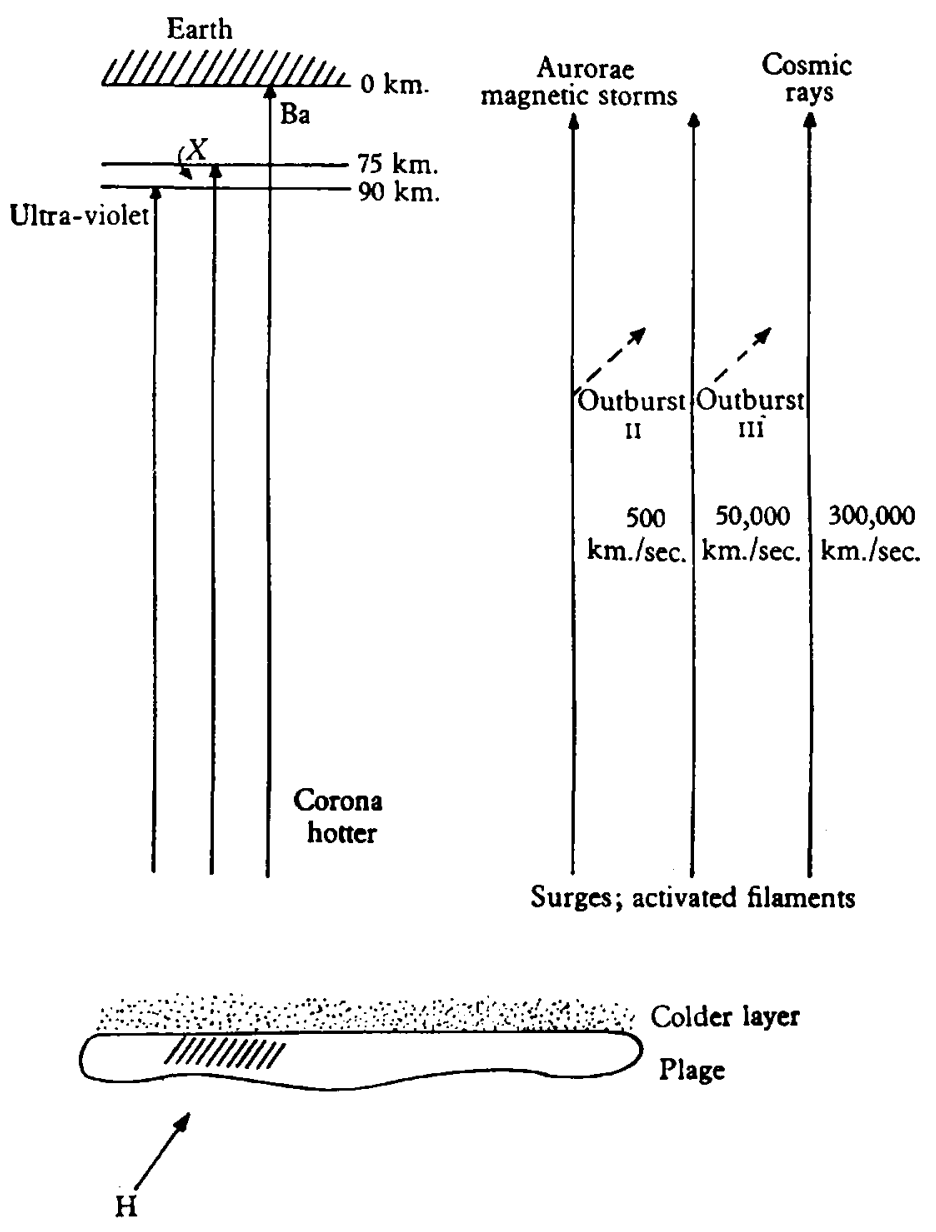

Fig. 6

Before a flare appears, there is already a special, perturbed temperature distribution over a certain solar area, which we call a plage. The apparition of a flare is due to the influence of a special disturbance, which might be a local magnetic field $H$ coming from inside the Sun. Apparently this produces electric currents, which then are amplified by the mechanism of Dr Unsöld till high temperatures are reached, accompanied by influences on the surroundings and by emission of radiation and corpuscules. 